\title{
Inferences From Dysregulated Long Non-Coding RNA-Mediated Competing Endogenous RNAs in Various Chemotherapy Drugs and Evaluation of Drug Response in Breast Cancer
}

\author{
Ziwen Zhang \\ Tumor Hospital of Harbin Medical University \\ Dongbo Li \\ Tumor Hospital of Harbin Medical University \\ Han Zhang \\ Tumor Hospital of Harbin Medical University \\ Qi Qin \\ Tumor Hospital of Harbin Medical University \\ Qingyuan Zhang ( $\square$ zqyhmu1965@163.com ) \\ Tumor Hospital of Harbin Medical University
}

\section{Primary research}

Keywords: Long non-coding RNA, Drug response, Competing endogenous RNAs, Breast cancer, Individualized treatment.

Posted Date: August 28th, 2020

DOI: https://doi.org/10.21203/rs.3.rs-65590/v1

License: (c) (i) This work is licensed under a Creative Commons Attribution 4.0 International License.

Read Full License 


\section{Abstract}

Backgroud: Differences in individual drug response, especially drug resistance, present an obstacle to the treatment of breast cancer (BRCA). Thus, the ability to predict drug response would contribute to developing novel treatment strategies. Accumulating evidence have suggested that tumor molecular profiles and drug response data provide opportunities and challenges for the discovery of new molecular characteristics and mechanisms of drug response in BRCA.

Methods: In the present study, an integrated pipeline was developed to explore drug response-related long non-coding RNA (IncRNA)-mediated competing endogenous RNAs (ceRNAs) motifs in BRCA.

Results: Drug response-specific ceRNAs indicated that IncRNAs play an essential role in various drug treatments for BRCA. Several key drug-resistant and -sensitive dysregulated ceRNAs were identified in Adriamycin, Cytoxan, and Tamoxifen. The interactions in these ceRNAs showed strong correlations in BRCA. Most drug response-related dysregulated ceRNAs were only present in one kind of drug. A number of drug response-related ceRNAs presented diverse dysregulation patterns. We also extracted some key drug response-related IncRNAs, such as HCP5 and FAM182A. These IncRNAs were associated with certain cancer hallmarks and survival in BRCA.

Conclusions: Ultimately, understanding the underlying IncRNA-mediated ceRNAs in drug responses will facilitate improved individual reactions to chemotherapy and overall outcomes of BRCA treatment.

\section{Introduction}

Among female cancer patients, breast cancer (BRCA) is the most common cancer and the second leading cause of cancer death after lung cancer [1]. The risk factors for breast cancer include certain menstrual and reproductive factors, postmenopausal obesity, use of hormone replacement therapy, family history of the disease, and carrying high-penetrance mutations [2]. Although the incidence rate of BRCA has increased slightly in recent years, the overall death rate continues to decline. This decrease in BRCA mortality could be accelerated by expanding access to high-quality prevention, early detection, and treatment services to all women. At present, surgical resection and chemotherapy are the two major modes of treatment for BRCA [3, 4]. Remarkably, different drug responses to BRCA chemotherapeutic drugs lead to diverse prognoses.

Numerous studies have shown that universal resistance to chemotherapeutic drugs is the most significant obstacle to successful BRCA treatment[5-7]. Current first-line chemotherapeutic drugs include Cytoxan, Adriamycin, and Tamoxifen. Cytoxan is an alkylating agent, which kills cells nonspecifically via chemical reactions with DNA and RNA molecules [8]. Adriamycin is a major chemotherapeutic drug that has been widely applied to BRCA patients [9]. Tamoxifen is often used in estrogen receptor alpha-positive BRCA patients, where it competitively blocks E2-binding in order to prevent co-activator-binding pocket formation and inhibit cell proliferation [10]. Although these chemotherapeutic drugs are all effective treatments for BRCA, acquired drug resistance frequently restricts their curative effects and leads to 
failure in the clinic. However, most existing studies on chemotherapeutic drug resistance focus on coding genes.

Long non-coding RNAs (IncRNAs) are an important type of non-coding RNAthat lack the ability to encode proteins. An increasing number of studies have found that IncRNAs play essential roles in the occurrence and development of diseases, including many kinds of cancers [11-13]. Regarding tumor drug resistance, IncRNAs also are emerging as key regulators of chemotherapeutic drug responses[14]. For example, IncRNA MALAT1 modulates the chemoresistance of Docetaxel-resistant lung adenocarcinoma cells [15]. LncRNA HORAS5 mediates castration-resistant prostate cancer survival by activating the androgen receptor transcriptional program [16]. It is believed that IncRNAs participate in the process of drug resistance via competing endogenous RNA (ceRNA) motifs. CeRNAs are transcripts that crossregulate each other by competing for shared microRNAs (miRNAs) [17]. For instance, IncRNA UCA1 promotes gefitinib resistance through a ceRNA that targets FOSL2 by sponging miR-143 in non-small cell lung cancer [18]. LncRNA GSTM3TV2 upregulates LAT2 and OLR1 by competitively sponging let-7 to promote gemcitabine resistance in pancreatic cancer [19]. Thus, systematic identification and characterization of the roles of ceRNAs in chemotherapeutic drug responses are essential.

In the present work, an integrated computational approach was developed to identify multiple chemotherapeutic drug response-related ceRNAs. Several differentially expressed IncRNAs, genes, and miRNAs were discovered in Cytoxan-, Adriamycin-, and Tamoxifen-resistant and -sensitive breast cancer groups. Drug-resistant and -sensitive ceRNA networks were constructed for each type of drug in BRCA patients. These networks showed specific topological features and core modules. The distributions of interactions were similar. Based on these ceRNAs, we identified several important IncRNAs that were related to drug response in BRCA. These drug response-related IncRNAs were associated with cancer hallmarks and thus may potentially serve as effective prognostic biomarkers in BRCA. In summary, this study found that IncRNA-mediated, drug response-specific ceRNAs play essential roles in BRCA.

\section{Materials And Methods}

\section{Collection of IncRNA, gene, and miRNA expression profiles in BRCA}

The expression profiles of whole-genome transcripts, including coding genes, miRNAs, and IncRNAs, were downloaded from The Cancer Genome Atlas (TCGA;https://gdc.xenahubs.net/download/TCGABRCA.htseq_fpkm.tsv.gz). The dataset included 1102 BRCA tumor and 390 control samples. All IncRNAs, genes, and miRNAs that were not expressed in all samples were excluded. The minimum value of all samples was given to any remaining expression values of 0 . All expression values were transformed using $\log _{2}($ value+1). In addition, clinical information, including chemotherapeutic drugs, drug response, disease process, and survival, were also obtained. Considering the number of samples and clinical applications, only the data from BRCA patients treated with Cytoxan, Adriamycin and Tamoxifen were extracted for subsequent analysis. BRCA patients with "complete response" were considered as the drug- 
sensitive group. BRCA patients with "stable disease,"'clinical progressive disease," or "partial response" were considered as the drug-resistant group.

\section{Acquisition of experiment-verified miRNA-IncRNA and miRNA-gene interactions to construct ceRNA motifs}

In order to obtain accurate ceRNA motifs, experiment-verified miRNA-IncRNA and miRNA-gene interactions were collected. We downloaded the miRNA-IncRNA interactions from the RNA Association Interaction Database (RAID)v2.0, which contained experimental and computational prediction RNA-RNA and RNA-protein interactions from manual literature readings and other database resources. Only miRNAIncRNA interactions with strong experimental evidence were extracted [20]. Then, gene-miRNA interactions were acquired from miRTarBase 7.0, which is a public database that includes numerous miRNA-target interactions [21]. Based on these two types of interactions, candidate ceRNA motifs were constructed.

\section{Identification of drug response-associated dysregulated ceRNAs by integrating expression and candidate ceRNA interactions in BRCA}

Drug-resistant and -sensitive dysregulated ceRNAs for Cytoxan, Adriamycin, and Tamoxifen in BRCA were identified using an integrated pipeline. First, differentially expressed IncRNAs, miRNAs, and genes were identified between drug-resistant and -sensitive groups for diverse kinds of drugs. Second, Pearson correlation coefficients (PCCs) were calculated in the drug-resistant and -sensitive groups based on expression values of the gene-IncRNA, gene-miRNA, and miRNA-IncRNA pairs. Third, we filtered drug response-associated dysregulated ceRNAs from expression datasets based on the following rules: PCC (IncRNA-gene) $>0.2$; PCC (IncRNA-miRNA) $<-0.2$, and PCC (gene-miRNA) <-0.2, where PCC (IncRNA-gene), PCC (IncRNA-miRNA), and PCC (gene-miRNA) represent the Pearson correlation coefficients of IncRNAgene, IncRNA-miRNA, and gene-miRNA interactions, respectively, based on expression values. Finally, if the PCC difference between drug-resistant and -sensitive groups for any one kind of interaction(geneIncRNA, gene-miRNA, or miRNA-IncRNA) was greater than 0.3 , the specific ceRNA was considered as a drug response-related dysregulated ceRNA in BRCA.

\section{Construction, topological feature analysis, and core module identification of drug response-associated dysregulated ceRNA networks}

Cytoscape software 3.3.0 (http://www.cytoscape.org/)was used to construct and illustrate the dysregulated ceRNA regulatory networks. Node degree was analyzed by the built-in Network Analyzer tool in Cytoscape. The degree for a node corresponds to the number of its network neighbors. Core modules were extracted from each dysregulated drug response-related ceRNA network based on the ClusterOne package in Cytoscape. ClusterOne could identify tightly connected core modules and score them. The number of nodes and scores were considered as reference conditions for screening core modules.

\section{Functional and cancer hallmark analyses for drug response-associated dysregulated ceRNAs in BRCA}


The Enrich web based tool (http://amp.pharm.mssm.edu/Enrichr/) with default parameters was used to perform functional annotation of IncRNAs in drug response-associated dysregulated ceRNAs in BRCA [22]. Significantly enriched Gene Ontology $(G O)$ terms $(P<0.05)$ were obtained. Cancer hallmark-related $\mathrm{GO}$ terms were acquired from a previous study [23]. The genes in these terms were downloaded from GO using AmiGo (version: 2.5.12; http://amigo.geneontology.org/amigo) [24]. All cancer hallmark-related genes were used to obtain interactions with genes in drug response-associated dysregulated ceRNAs.

\section{Survival analysis for drug response-associated dysregulated ceRNAs in BRCA}

To evaluate the efficiency of drug response-associated dysregulated ceRNAs in predicting the survival of BRCA patients, an integrated risk model was constructed. Univariate Cox regression analysis was performed to demonstrate the associations between survival and expression levels of IncRNAs, genes, and miRNAs in drug response-associated dysregulated ceRNAs. The risk score for each BRCA patient was computed based on the linear combination of expression values weighted by the coefficient from the univariate Cox regression analysis:

$$
\text { Riskscore }=\sum_{i=1}^{N} \operatorname{Cox}_{i}^{*} \operatorname{Exp}(i)
$$

Where Cox $_{i}$ is the Cox regression coefficient of an IncRNA, gene, or miRNA node in a specific drug response-associated dysregulated ceRNA, N is the number of nodes in a ceRNA motif, and $\operatorname{Exp}(i)$ is the expression value of node i. All BRCA patients were divided into high-and low-risk groups based on the median risk score. The patients with high- and low-risk scores were expected to have poor and good survival outcomes, respectively. Kaplan-Meier survival analysis was performed for the two groups. The log-rank test was used to assess statistical significance $(P<0.05)$. R 3.6.2 statistical software(The $R$ Foundation, Vienna, Austria) was utilized for all analyses.

\section{Results}

\section{Differentially expressed key genes between drug-resistant and -sensitive BRCA patients treated with diverse kinds of chemotherapy drugs}

In order to characterize the molecular features in BRCA patients with diverse drug responses, we identified drug-resistant and -sensitive differentially expressed IncRNAs, genes, and miRNAs. In Cytoxan, Adriamycin, and Tamoxifen, the quantity of differentially expressed IncRNAs, genes, and miRNAs was variable (Figure 1A). We discovered that most of these differentially expressed molecules were downregulated. Some of these IncRNAs, genes, and miRNAs were differentially expressed in multiple kinds of chemotherapy drugs (Figure 1B). We identified 2446, 579, and 110 common drug responserelated IncRNAs, genes, and miRNAs, respectively, in three kinds of drugs. However, several of these common drug response-related IncRNAs, genes, and miRNAs showed different expression patterns (Figure 1C). For example, $9.73 \%, 8.63 \%$ and $12.73 \%$ of molecules showed opposite expression patterns 
among Cytoxan, Adriamycin, and Tamoxifen, respectively. We also focused on a number of key IncRNAs, which also displayed different dysregulated expression patterns (Figure 1D). LncRNA CTB-164N12.1 was upregulated in Cytoxan and Adriamycin but downregulated in Tamoxifen. Several other IncRNAs, including LINC01028, CTD-2128A3.2, and RP11-167H9.4,revealedsimilar dysregulated expression patterns. These results indicate that certain IncRNAs, genes, and miRNAs play essential roles in the drug responses of BRCA patients. Furthermore, several IncRNAs showed more complex regulatory patterns in BRCA drug responses.

\section{Specific features of drug-resistant and -sensitive dysregulated ceRNA networks}

An integrated computational pipeline was designed to identify drug-resistant and -sensitive dysregulated ceRNAs. We found 1574, 1630, and 2990 drug-resistant dysregulated ceRNAs in Cytoxan, Adriamycin and Tamoxifen, respectively. Furthermore, 48, 44, and 213 drug-sensitive dysregulated ceRNAs were identified in Cytoxan, Adriamycin and Tamoxifen, respectively (Figure 2A). In order to better describe drug responserelated dysregulated ceRNAs, drug-resistant and -sensitive dysregulated ceRNA networks were constructed for the aforementioned three drugs. The drug-resistant dysregulated ceRNA network of Tamoxifen had most edges and nodes (Figure 2B). Three drug-resistant dysregulated ceRNA networks showed scale-free topological features (Figure $2 \mathrm{C}$ and D). Only a small number of nodes in these networks had great degrees. This finding indicates that drug response-related dysregulated ceRNA networks are informative biological networks. We also extracted some core modules from the ceRNA networks of Cytoxan, Adriamycin, and Tamoxifen (Figure 2E). The core module in the Adriamycin network had 9 nodes (1 IncRNA, 1 miRNA, and 7 genes) and 15 edges. IGF2BP2-AS1 is an IncRNA located in the antisense chain of coding gene IGF2BP2. A previous study reported that IGF2BP2-AS1 is associated with survival in lung squamous cell carcinoma [25]. Accumulating evidence has also suggested a link between dysregulation of IGF2BP2 and cancer [26]. IGF2BP2 has been hypothesized to serve as an N6methyladenosine reader for the promotion of cancer stemness-like properties and pancreatic cancer pathogenesis [27].

\section{Evaluation ofpatterns in drug-resistant and -sensitive dysregulated ceRNAs}

We used expression correlations between any two molecules in each dysregulated ceRNA to evaluate the patterns of drug-resistant and -sensitive dysregulated ceRNA networks. We found that the co-expressed correlations between miRNA-IncRNA, miRNA-gene, and gene-IncRNA interactions in both drug-resistant and -sensitive dysregulated ceRNA networks showed similar distributions (Figure 3A). PCC differences between drug-resistant and -sensitive groups for any one kind of interaction (gene-IncRNA, gene-miRNA,or miRNA-IncRNA) were also analyzed. We discovered that most of these differences were concentrated between 0.2 and 0.6 in the three kinds of drugs (Figure 3B). In addition, we analyzed the number of dysregulated interactions. Among the three kinds of drug-resistant dysregulated ceRNAs, two dysregulated interactions were most commonly observed. For example, there were 211, 746 and 617 ceRNAs with one, two, and three dysregulated interactions, respectively, in Adriamycin-resistant ceRNAs. In drug-sensitive dysregulated ceRNAs, most ceRNAs only displayed one dysregulated interaction (Figure 
3C, D). These results indicate that our method was accurate and reliable for identifying drug-resistant and -sensitive dysregulated ceRNAs.

\section{Common and specific drug-resistant and -sensitive dysregulated ceRNAs in BRCA}

We also evaluated common and specific features of drug-resistant and -sensitive dysregulated ceRNAs. More than $90 \%$ of drug response-related ceRNAs were only dysregulated in one kind of drug (Figure 4A). However, a small number of ceRNAs were dysregulated in two or all three kinds of drugs. For example, ceRNA UCP1/miR-130b-3p/RMST were dysregulated in all three drugs (Figure 4B), and ceRNA F0X01/miR-15a-5p/CLRN1-AS1 were dysregulated in two kinds of drugs. Although some drug responserelated ceRNAs were dysregulated in multiple kinds of drugs, their dysregulation patterns were diverse. For example, ceRNA UCA1/miR-16-5p/BACE1 were dysregulated in the three kinds of drugs and all displayed three dysregulated interactions (Figure 4C). More ceRNAs showed different dysregulation patterns than similar ones. Dysregulated interactions were diverse for ceRNA OTX2-AS1/miR-16$5 p / T P P P 3$ in all three kinds of drugs. In addition, we analyzed the frequency of IncRNAs, genes, and miRNAs to identify key molecules (Figure 4D). For example, key IncRNAs like HCP5, FAM182A, and NPHP3-AS1 were discovered. Newly identifiedkey genes included PTEN, HMGA2, and CCND1. We also found that $26.28 \%, 61.05 \%$, and $27.51 \%$ of IncRNAs, miRNAs, and genes occurred in three, two, and one kind(s) of drugs, respectively (Figure 4E). Notably, IncRNA HCP5, which had the highest frequency, was only dysregulated in Tamoxifen (Figure 4F). A number of other IncRNAs with high frequency were dysregulated in multiple drugs.

\section{Cancer hallmarks in drug-resistant and -sensitive-dysregulated ceRNAs}

In order to depict the roles of drug-resistant and -sensitive dysregulated ceRNAs, we performed a functional analysis of IncRNAs. These IncRNAs were enriched in several essential GO terms, including regulation of biosynthetic processes, response to gonadotropin, polyphosphate metabolic processes, and regulation of inositol phosphate biosynthetic processes (Figure 5A). Some of these GO enrichment terms were related to the development and progression of BRCA. For example, gonadotropin is a glycoprotein hormone that regulates the gonadal development of vertebrates as well as promotes the production and secretion of sex hormones. A large number of studies have reported that BRCA is well known for being strongly influenced by female steroids [28-30]. Inositol phosphate is also thought to play an essential role in BRCA [31]. In addition, we explored the relationships between drug-resistant and -sensitive dysregulated ceRNAs and cancer hallmarks. We found that some cancer hallmarks, such as tissue invasion and metastasis, self-sufficiency, insensitivity to antigrowth signals, and evasion of apoptosis, were associated with multiple genes in drug-resistant and -sensitive dysregulated ceRNAs (Figure 5B). Together, these results indicate that drug-resistant and -sensitive dysregulated ceRNAs have specific functions in BRCA.

\section{Efficacy of drug-resistant and -sensitive dysregulated ceRNAs as prognostic biomarkers in BRCA}

Finally, we utilized a comprehensive computational method to evaluate the prognosis of drug-resistant and -sensitive dysregulated ceRNAs. Each drug type displayed a different percentage of survival-related 
drug-resistant and -sensitive dysregulated ceRNAs (Figure 6A). For example, we observed that $86.36 \%$ of drug sensitive-related dysregulated ceRNAs in Cytoxan were related to survival (Figure 6B). The HR values for genes, IncRNAs, and miRNAs in a given dysregulated ceRNA were diverse (Figure 6C). These values represent relative protection and risk for molecule survival. We also evaluated the survival of some key drug-resistant and -sensitive dysregulated ceRNAs (Figure 6D). Dysregulated ceRNA PURA/LINC02120/miR-15a-5p was significantly associated with survival $(P<0.001)$. Dysregulated ceRNA MTDH/LINC00092/miR-542-3p was also significantly associated with survival $(P<0.001)$. In addition, BRCA patients with high-risk scores showed poorer prognoses. All of these results suggest that drugresistant and -sensitive dysregulated ceRNAs could serve as effective prognostic biomarkers in BRCA.

\section{Discussion}

Chemotherapy is currently the primary and most effective treatment for various cancers, including BRCA, but its efficacy is limited by individual differences in drug response. Drug resistance is one of the notable reasons for BRCA treatment failure. Thus, an understanding of individual drug responses for BRCA patients is urgently needed to improve BRCA treatment. Recent studies have indicated that a number of IncRNAs play essential roles in drug response. In the present work, we identified drug response-related IncRNAs based on ceRNA motifs in BRCA. An integrated and computational pipeline was designed to identify drug response-related ceRNAs for multiple drug types according to IncRNA, miRNA, and gene expression levels in BRCA. Thus, we extracted a number of drug-resistant and -sensitive ceRNAs for Adriamycin, Cytoxan, and Tamoxifen in BRCA. Drug response-related ceRNAs displayed diverse dysregulation patterns in multiple drugs. Several key IncRNAs, miRNAs, and genes, such as FAM182A, miR-155-5p, and PTEN, were identified based on these dysregulated drug response-related ceRNAs. These ceRNAs were also associated with certain notable cancer hallmarks and thus have the potential to serve as prognostic biomarkers in BRCA.

CeRNAsare an important regulatory mechanism for IncRNAs in cancer [32]. In 2011, Salmena et al. proposed the ceRNA hypothesis on bona fide communications between coding and noncoding RNAs. Recent evidence has highlighted the crucial regulatory roles of ceRNA networks in many kinds of cancers $[17,33,34]$. The functions of ceRNAs in BRCA were also investigated. For example, FOXO1 3'UTR may function as a miRNA inhibitor for modulating metastasis [35], and IncRNA PVT1 is thought to form ceRNA with miR-200 in BRCA [36]. CeRNAs also play specific roles in BRCA drug resistance. LncRNA NONHSAT101069 was upregulated in BRCA tissues and promoted epirubicin resistance via regulation of the NONHSAT101069/miR-129-5p/Twist1 axis, highlighting its potential as an oncogene and therapeutic biomarker for BRCA [37]. The IncRNA regulator of reprogramming promotes the progression of BRCA and decreases sensitivity to rapamycin through miR-194-3p targeting of MECP2 [38]. In our study, we focused on three kinds of drugs, including Adriamycin, Cytoxan, and Tamoxifen,for BRCA. In these three drug types, several drug-resistant and -sensitive dysregulated ceRNAs were identified and analyzed. Thus, our work provides a number of candidate drug response-related ceRNAs in BRCA. 
We further analyzed drug-specific dysregulations for theceRNAs in BRCA. Most of them were only dysregulated in one kind of drug. This result indicates that IncRNAs may exert their diverse roles through ceRNA to influence multiple types of drug responses in BRCA. Although a small number of drug responserelated ceRNAs were found to be dysregulated in multiple kinds of drugs, they still showed diverse dysregulated patterns. For example, the interactions of drug response-related ceRNAs miR-16-5p/OTX2AS1/BACE1 were different. In Adriamycin, the interaction between miR-16-5p and OTX2-AS1 was impacted. In Cytoxan, two interactions (miR-16-5p and OTX2-AS1 as well as OTX2-AS1 and TPPP3) were dysregulated. In Tamoxifen, two interactions were also disrupted (miR-16-5p and OTX2-AS1, miR-16-5p and TPPP3). These different dysregulated interactions contribute to drug-specific responses in BRCA ceRNAs.

In addition, we identified several key drug response-related IncRNAs, miRNAs, and genes. For example, a number of key IncRNAs like HCP5, LINC01085, FAM182A, LINC01559, NPHP3-AS1, and FGF13-AS1 were identified. Some of these IncRNAs have been reported to be associated with drug response. For example, IncRNA HCP5 promoted fatty acid oxidation through the miR-3619-5p/AMPK/PGC1/CEBPB axis to promote stemness and chemoresistance in gastric cancer, indicating that targeting HCP5 was a novel approach to enhancing chemotherapy efficacy for gastric cancer. In our work, we found that HCP5 was present in 339 drug response-related dysregulated ceRNAs, all of which were in Tamoxifen. Thus, this result indicates that IncRNA HCP5 maybe a key drug response-related IncRNA for Tamoxifen resistance in BRCA.

\section{Conclusion}

In the present study, a number of key drug response-related ceRNAs were identified and characterized in BRCA. Our work provides novel insights for leveraging publicly available molecular data to evaluate clinical drug responses and contribute to personalized cancer medicine. We propose that IncRNAmediated ceRNAs have the potential to serve as biomarkers for drug resistance, thereby providing an avenue for improved individual treatment of BRCA.

\section{Declarations}

\section{Authors' contributions}

ZQY conceived and designed the experiments. ZZW, LDB, ZH and QQ analyzed data. ZZW wrote this manuscript. All authors read and approved the final manuscript.

\section{Acknowledgements}

Not applicable.

\section{Competing interests}


The authors declare that they have no competing interests.

Availability of data and materials

Not applicable.

Consent for publication

Not applicable.

Ethics approval and consent to participate

Not applicable.

Funding

Not applicable.

\section{References}

1. DeSantis CE, Ma J, Gaudet MM, Newman LA, Miller KD, Goding Sauer A, Jemal A, Siegel RL. Breast cancer statistics, 2019. CA: a cancer journal for clinicians. 2019; 69: 438-51.

2. Shu X, Bao J, Wu L, Long J, Shu XO, Guo X, Yang Y, Michailidou K, Bolla MK, Wang Q, Dennis J, Andrulis IL, Castelao JE, Dork T, Gago-Dominguez M, Garcia-Closas M, Giles GG, Lophatananon A, Muir K, Olsson H, Rennert G, Saloustros E, Scott RJ, Southey MC, Pharoah PDP, Milne RL, Kraft P, Simard J, Easton DF, Zheng W. Evaluation of associations between genetically predicted circulating protein biomarkers and breast cancer risk. International journal of cancer. 2020; 146: 2130-8.

3. Bale R, Putzer D, Schullian P. Local Treatment of Breast Cancer Liver Metastasis. Cancers. 2019; 11.

4. Waks AG, Winer EP. Breast Cancer Treatment: A Review. Jama. 2019; 321: 288-300.

5. Hanker AB, Sudhan DR, Arteaga CL. Overcoming Endocrine Resistance in Breast Cancer. Cancer cell. 2020; 37: 496-513.

6. Saatci O, Kaymak A, Raza U, Ersan PG, Akbulut O, Banister CE, Sikirzhytski V, Tokat UM, Aykut G, Ansari SA, Dogan HT, Dogan M, Jandaghi P, Isik A, Gundogdu F, Kosemehmetoglu K, Dizdar O, Aksoy S, Akyol A, Uner A, Buckhaults PJ, Riazalhosseini Y, Sahin O. Targeting lysyl oxidase (LOX) overcomes chemotherapy resistance in triple negative breast cancer. Nature communications. 2020; 11: 2416.

7. Zhang J, Meng H, Zhang M, Zhang C, Huang M, Yan C, Wang Z, Hou L, Yang L, Ling R. Regulation of docetaxel chemosensitivity by NR2F6 in breast cancer. Endocrine-related cancer. 2020.

8. Cleator S, Tsimelzon A, Ashworth A, Dowsett M, Dexter T, Powles T, Hilsenbeck S, Wong H, Osborne CK, O'Connell P, Chang JC. Gene expression patterns for doxorubicin (Adriamycin) and cyclophosphamide (cytoxan) (AC) response and resistance. Breast cancer research and treatment. 2006; 95: 229-33. 
9. Chen Z, Pan T, Jiang D, Jin L, Geng Y, Feng X, Shen A, Zhang L. The IncRNA-GAS5/miR-221-3p/DKK2 Axis Modulates ABCB1-Mediated Adriamycin Resistance of Breast Cancer via the Wnt/beta-Catenin Signaling Pathway. Molecular therapy Nucleic acids. 2020; 19: 1434-48.

10. Flach KD, Periyasamy M, Jadhav A, Dorjsuren D, Siefert JC, Hickey TE, Opdam M, Patel H, Canisius S, Wilson DM, 3rd, Donaldson Collier M, Prekovic S, Nieuwland M, Kluin RJC, Zakharov AV, Wesseling J, Wessels LFA, Linn SC, Tilley WD, Simeonov A, Ali S, Zwart W. Endonuclease FEN1 Coregulates ERalpha Activity and Provides a Novel Drug Interface in Tamoxifen-Resistant Breast Cancer. Cancer research. 2020; 80: 1914-26.

11. Esposito R, Bosch N, Lanzos A, Polidori T, Pulido-Quetglas C, Johnson R. Hacking the Cancer Genome: Profiling Therapeutically Actionable Long Non-coding RNAs Using CRISPR-Cas9 Screening. Cancer cell. 2019; 35: 545-57.

12. Evans JR, Feng FY, Chinnaiyan AM. The bright side of dark matter: IncRNAs in cancer. The Journal of clinical investigation. 2016; 126: 2775-82.

13. Huang QY, Liu GF, Qian XL, Tang LB, Huang QY, Xiong LX. Long Non-Coding RNA: Dual Effects on Breast Cancer Metastasis and Clinical Applications. Cancers. 2019; 11.

14. Leucci E. Cancer development and therapy resistance: spotlights on the dark side of the genome. Pharmacology \& therapeutics. 2018; 189: 22-30.

15. Chen J, Liu X, Xu Y, Zhang K, Huang J, Pan B, Chen D, Cui S, Song H, Wang R, Chu X, Zhu X, Chen L. TFAP2C-Activated MALAT1 Modulates the Chemoresistance of Docetaxel-Resistant Lung Adenocarcinoma Cells. Molecular therapy Nucleic acids. 2019; 14: 567-82.

16. Parolia A, Venalainen E, Xue H, Mather R, Lin D, Wu R, Pucci P, Rogalski J, Evans JR, Feng F, Collins CC, Wang Y, Crea F. The long noncoding RNA HORAS5 mediates castration-resistant prostate cancer survival by activating the androgen receptor transcriptional program. Molecular oncology. 2019; 13: 1121-36.

17. Qi X, Zhang DH, Wu N, Xiao JH, Wang X, Ma W. ceRNA in cancer: possible functions and clinical implications. Journal of medical genetics. 2015; 52: 710-8.

18. Chen X, Wang Z, Tong F, Dong X, Wu G, Zhang R. IncRNA UCA1 Promotes Gefitinib Resistance as a ceRNA to Target FOSL2 by Sponging miR-143 in Non-small Cell Lung Cancer. Molecular therapy Nucleic acids. 2020; 19: 643-53.

19. Xiong G, Liu C, Yang G, Feng M, Xu J, Zhao F, You L, Zhou L, Zheng L, Hu Y, Wang X, Zhang T, Zhao Y. Long noncoding RNA GSTM3TV2 upregulates LAT2 and OLR1 by competitively sponging let-7 to promote gemcitabine resistance in pancreatic cancer. Journal of hematology \& oncology. 2019; 12: 97.

20. Yi Y, Zhao Y, Li C, Zhang L, Huang H, Li Y, Liu L, Hou P, Cui T, Tan P, Hu Y, Zhang T, Huang Y, Li X, Yu J, Wang D. RAID v2.0: an updated resource of RNA-associated interactions across organisms. Nucleic acids research. 2017; 45: D115-D8.

21. Chou CH, Shrestha S, Yang CD, Chang NW, Lin YL, Liao KW, Huang WC, Sun TH, Tu SJ, Lee WH, Chiew MY, Tai CS, Wei TY, Tsai TR, Huang HT, Wang CY, Wu HY, Ho SY, Chen PR, Chuang CH, Hsieh 
PJ, Wu YS, Chen WL, Li MJ, Wu YC, Huang XY, Ng FL, Buddhakosai W, Huang PC, Lan KC, Huang CY, Weng SL, Cheng YN, Liang C, Hsu WL, Huang HD. miRTarBase update 2018: a resource for experimentally validated microRNA-target interactions. Nucleic acids research. 2018; 46: D296-D302.

22. Kuleshov MV, Jones MR, Rouillard AD, Fernandez NF, Duan Q, Wang Z, Koplev S, Jenkins SL, Jagodnik KM, Lachmann A, McDermott MG, Monteiro CD, Gundersen GW, Ma'ayan A. Enrichr: a comprehensive gene set enrichment analysis web server 2016 update. Nucleic acids research. 2016; 44: W90-7.

23. Plaisier CL, Pan M, Baliga NS. A miRNA-regulatory network explains how dysregulated miRNAs perturb oncogenic processes across diverse cancers. Genome research. 2012; 22: 2302-14.

24. Carbon S, Ireland A, Mungall CJ, Shu S, Marshall B, Lewis S, Ami GOH, Web Presence Working G. AmiGO: online access to ontology and annotation data. Bioinformatics. 2009; 25: 288-9.

25. Liu B, Chen Y, Yang J. LncRNAs are altered in lung squamous cell carcinoma and lung adenocarcinoma. Oncotarget. 2017; 8: 24275-91.

26. Cao J, Mu Q, Huang H. The Roles of Insulin-Like Growth Factor 2 mRNA-Binding Protein 2 in Cancer and Cancer Stem Cells. Stem cells international. 2018; 2018: 4217259.

27. Hu X, Peng WX, Zhou H, Jiang J, Zhou X, Huang D, Mo YY, Yang L. IGF2BP2 regulates DANCR by serving as an N6-methyladenosine reader. Cell death and differentiation. 2020; 27: 1782-94.

28. Kim J, Turan V, Oktay K. Long-Term Safety of Letrozole and Gonadotropin Stimulation for Fertility Preservation in Women With Breast Cancer. The Journal of clinical endocrinology and metabolism. 2016; 101: 1364-71.

29. Schuler-Toprak S, Treeck O, Ortmann O. Human Chorionic Gonadotropin and Breast Cancer. International journal of molecular sciences. 2017; 18.

30. Subramani R, Nandy SB, Pedroza DA, Lakshmanaswamy R. Role of Growth Hormone in Breast Cancer. Endocrinology. 2017; 158: 1543-55.

31. Morgan K, Meyer C, Miller N, Sims AH, Cagnan I, Faratian D, Harrison DJ, Millar RP, Langdon SP. $\mathrm{GnRH}$ receptor activation competes at a low level with growth signaling in stably transfected human breast cell lines. BMC cancer. 2011; 11: 476.

32. Salmena L, Poliseno L, Tay Y, Kats L, Pandolfi PP. A ceRNA hypothesis: the Rosetta Stone of a hidden RNA language? Cell. 2011; 146: 353-8.

33. Abdollahzadeh R, Daraei A, Mansoori Y, Sepahvand M, Amoli MM, Tavakkoly-Bazzaz J. Competing endogenous RNA (ceRNA) cross talk and language in ceRNA regulatory networks: A new look at hallmarks of breast cancer. Journal of cellular physiology. 2019; 234: 10080-100.

34. Shuwen H, Qing Z, Yan Z, Xi Y. Competitive endogenous RNA in colorectal cancer: A systematic review. Gene. 2018; 645: 157-62.

35. Yang J, Li T, Gao C, Lv X, Liu K, Song H, Xing Y, Xi T. FOXO1 3'UTR functions as a ceRNA in repressing the metastases of breast cancer cells via regulating miRNA activity. FEBS letters. 2014; 588: 3218-24. 
36. Conte F, Fiscon G, Chiara M, Colombo T, Farina L, Paci P. Role of the long non-coding RNA PVT1 in the dysregulation of the ceRNA-ceRNA network in human breast cancer. PloS one. 2017; 12: e0171661.

37. Yao N, Fu Y, Chen L, Liu Z, He J, Zhu Y, Xia T, Wang S. Long non-coding RNA NONHSAT101069 promotes epirubicin resistance, migration, and invasion of breast cancer cells through NONHSAT101069/miR-129-5p/Twist1 axis. Oncogene. 2019; 38: 7216-33.

38. Zhou Q, Guo J, Huang W, Yu X, Xu C, Long X. Linc-ROR promotes the progression of breast cancer and decreases the sensitivity to rapamycin through miR-194-3p targeting MECP2. Molecular oncology. 2020.

\section{Figures}



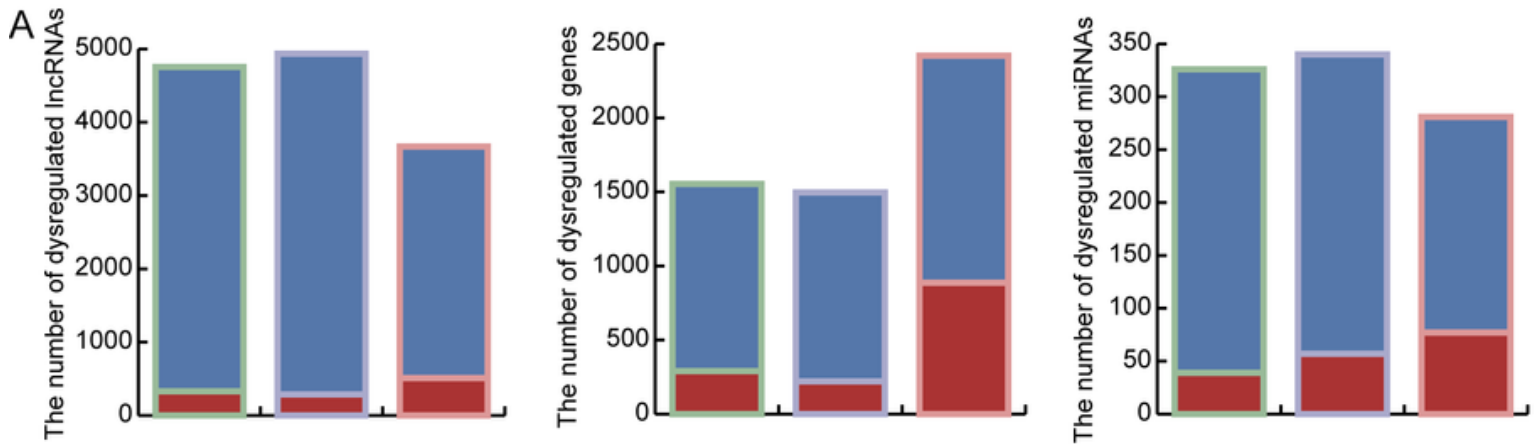

Up-regulation

Down-regulation

Adriamycin

Cytoxan

Tamoxifen

B

IncRNA

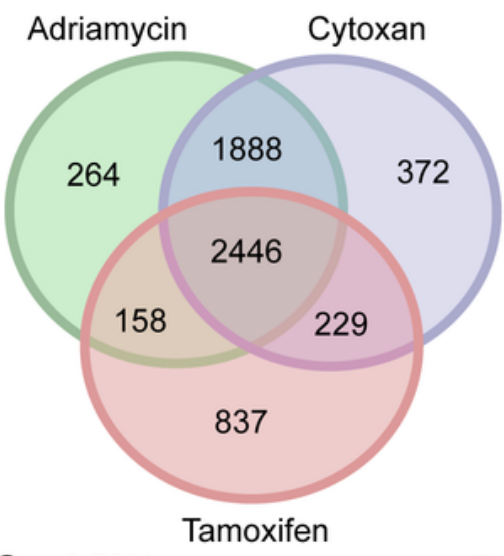

C $\quad 9.73 \%$

$2.37 \%$

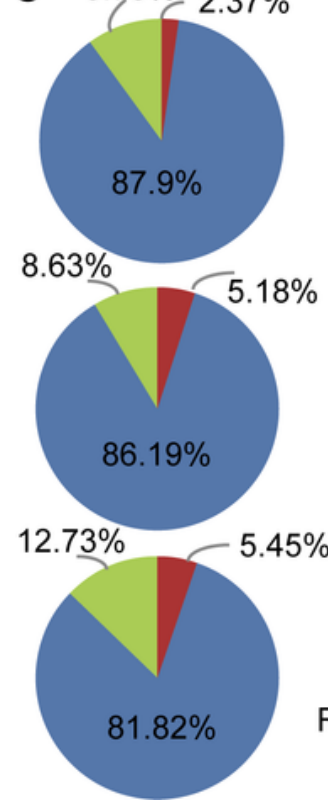

D

CTB-164N12.1

LINC01028

CTD-2128A3.2

RP11-167H9.4

LINC01234

LINC01807

LINC00491

C5orf66-AS2

STX18-IT1

MIR137HG

RP11-26L20.3

LINC01317

LINC01887

RP11-433A10.3

LBX1 AS 1
Gene

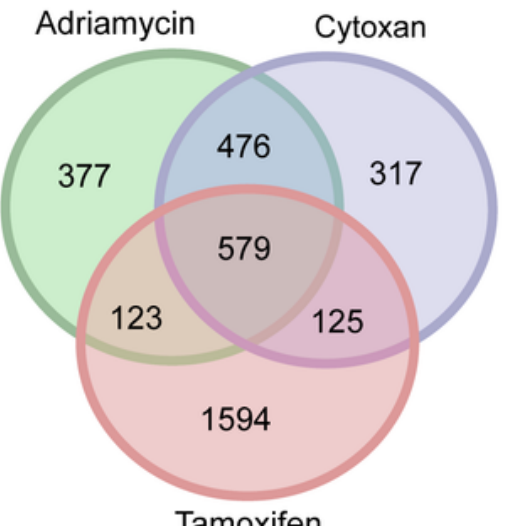

Tamoxifen
miRNA

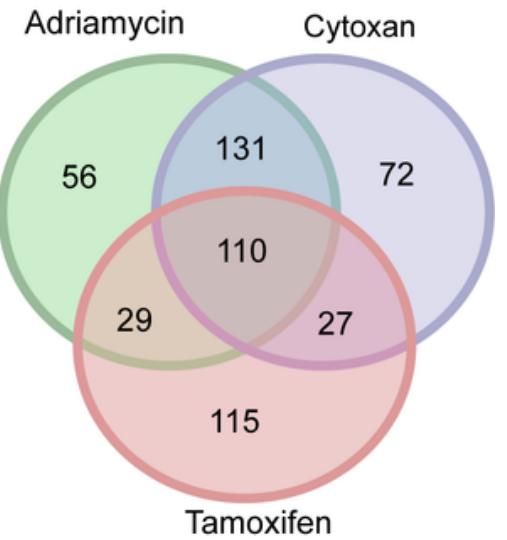

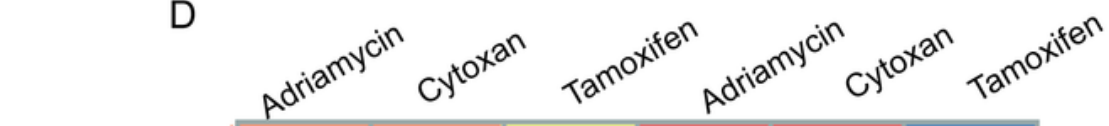

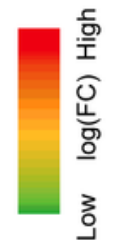

\section{Figure 1}

Differentially expressed key genes between drug-resistant and -sensitive BRCA patients treated with diverse kinds of chemotherapy drugs. (A) Bar plots showing the numbers of dysregulated IncRNAs. Red and blue represent up and downregulated IncRNAs, respectively. Green, purple, and pink represent Adriamycin, Cytoxan, and Tamoxifen, respectively. (B) Venn diagrams showing the intersections of dysregulated IncRNAs, genes, and miRNAs among Adriamycin, Cytoxan, and Tamoxifen. (C) Pie charts 
showing the percentages of dysregulated IncRNAs in one, two,orall three types of drugs. (D) A heat map showing the expression values and dysregulated patterns in each kind of drug.

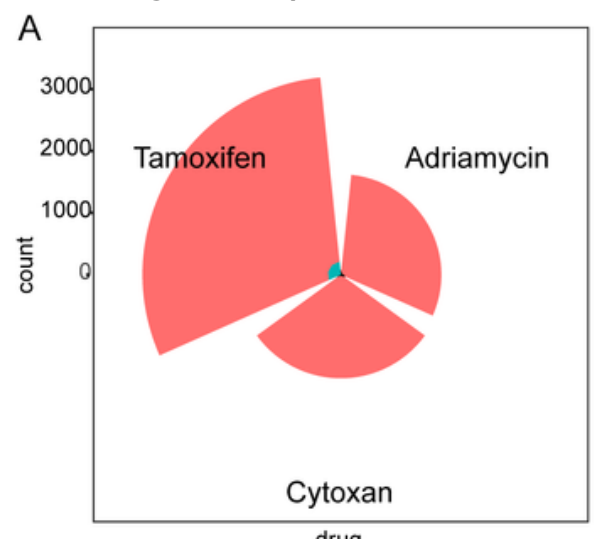

Drug response $\square$ resistant $\square$ sensitive
B
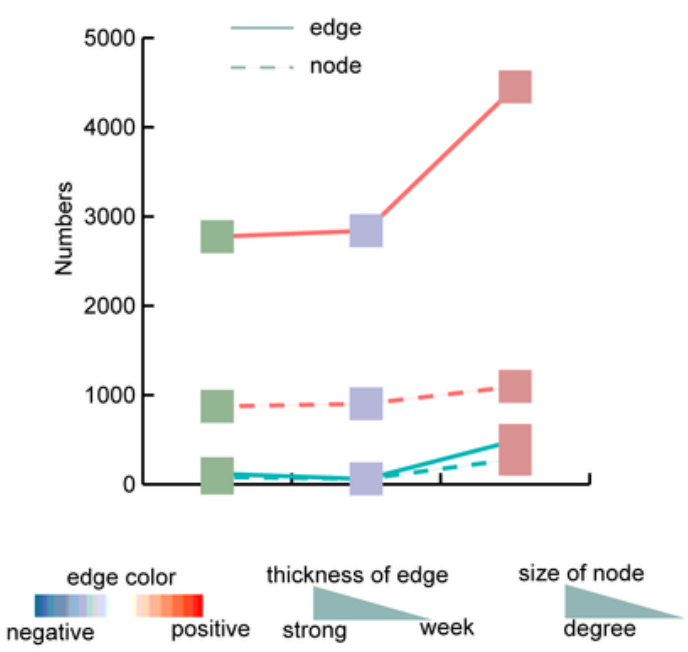

Dysregulated Adriamycin-related ceRNAs

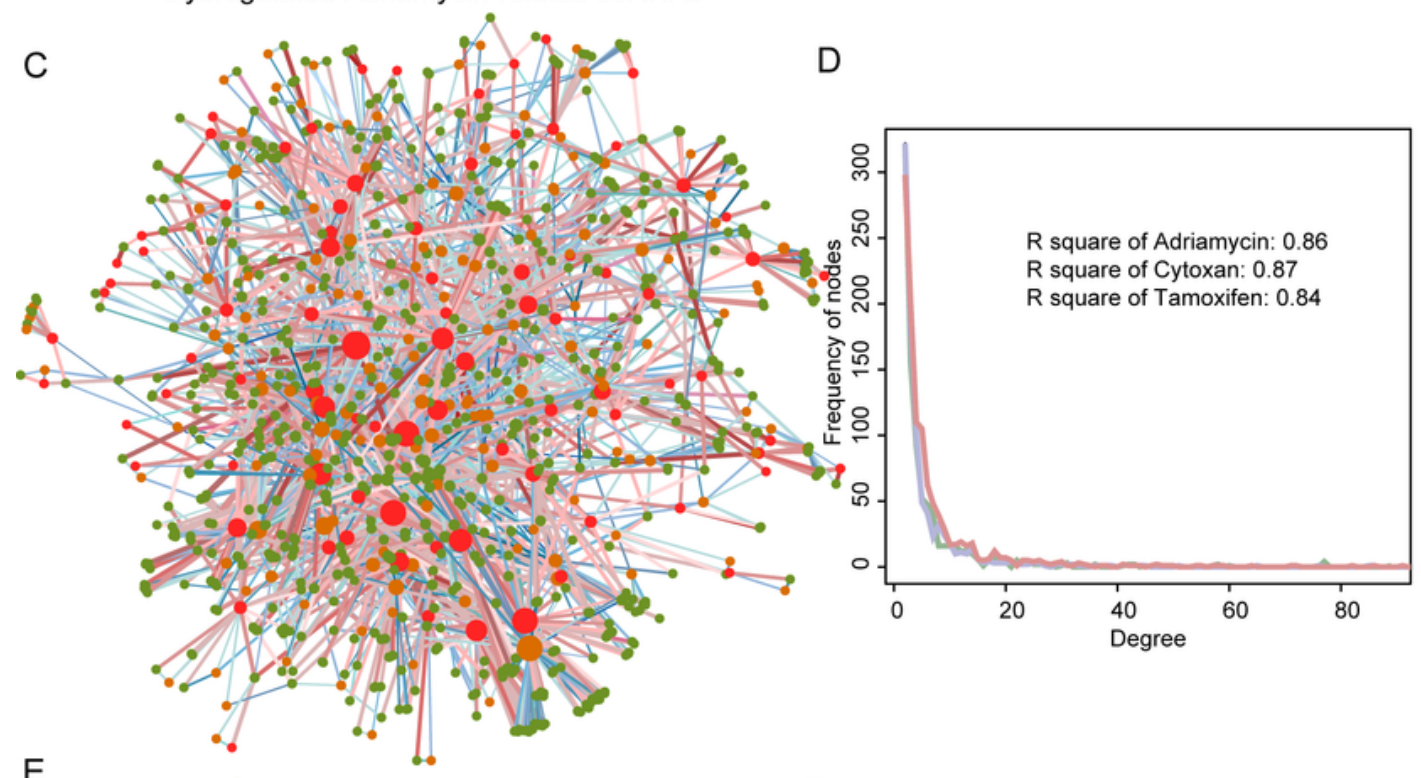

$\mathrm{E}$

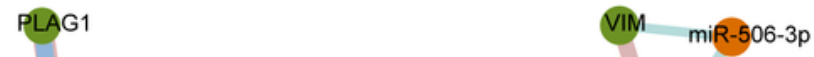

TGFBRAP1

PLI

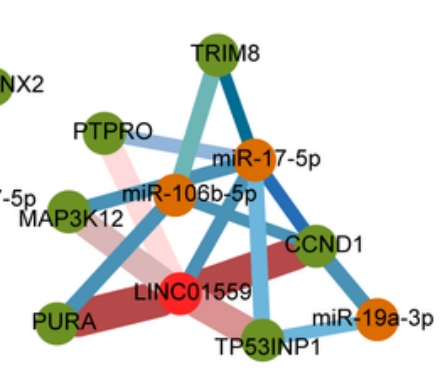

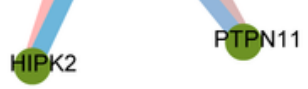

Adriamycin

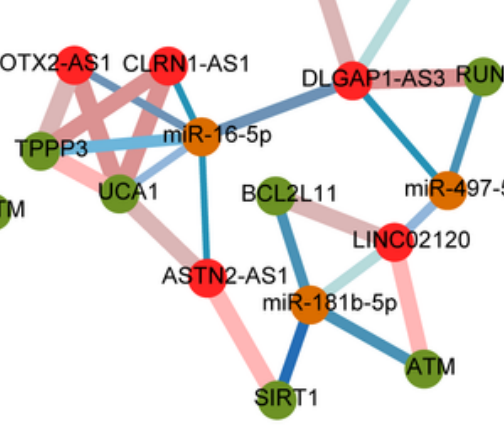

Cytoxan

Tamoxifen

Figure 2

Specific features of drug-resistant and -sensitive dysregulated ceRNA networks. (A) A rose diagram showing the drug-resistant (purple) and -sensitive (blue) dysregulated ceRNAs. (B) Line charts showing the numbers of nodes and edges in dysregulated ceRNA networks. (C) Drug-resistant dysregulated ceRNA 
networks. The size of each node indicates the degree, and the red and blue edges represent up and downregulated interactions. (D) A line plot showing the degree distribution of drug-resistant dysregulated ceRNA networks in the multiple types of drugs. (E) Core modules extracted from drug-resistant dysregulated ceRNA networks in the multiple types of drugs.
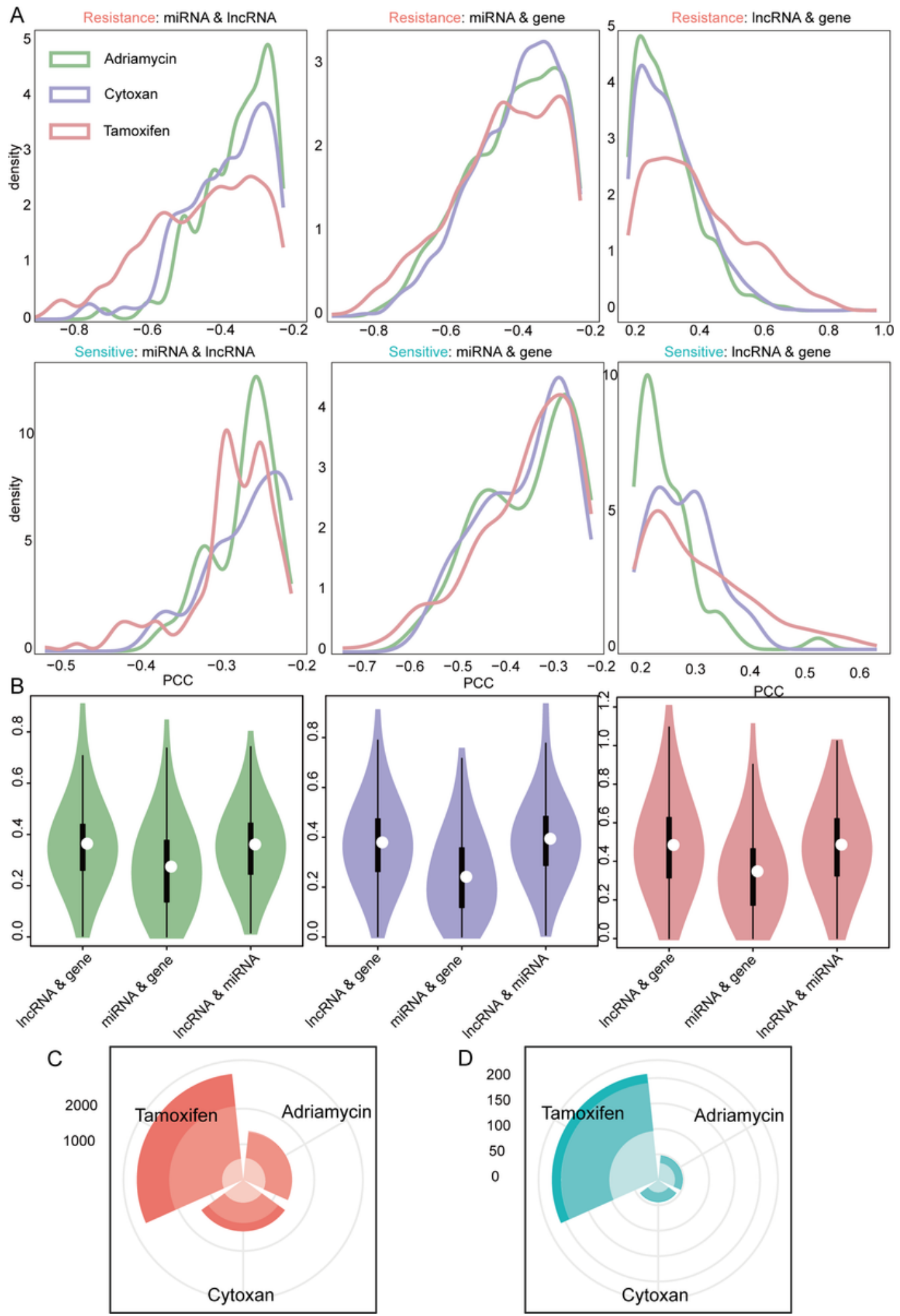

\section{Figure 3}


Evaluation of patterns in drug-resistant and -sensitive dysregulated ceRNAs. (A) Density curves showing the correlation between two molecules in Adriamycin, Cytoxan, and Tamoxifen. (B) Violin plots showing the correlation between each interaction in the multiple types of drugs. (C) Rose diagrams of the observed one, two, or three dysregulated interactions in Adriamycin-resistant ceRNAs.
A 48
442
$0.85 \%$ $-7.81 \%$

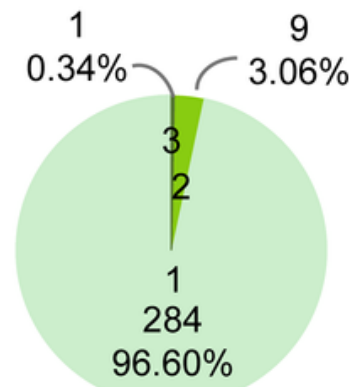
B FOXO1/miR-15a-5p/CLRN1-AS1

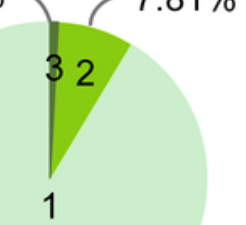
5166
$91.34 \%$
$96.60 \%$
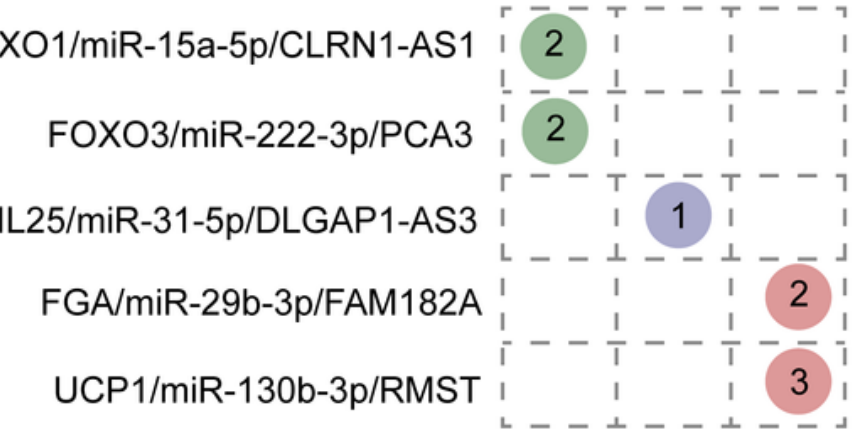
C

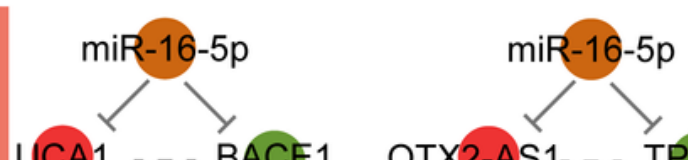
UCA1 - - BACE1 OTX2-AS1 - - TPPP3
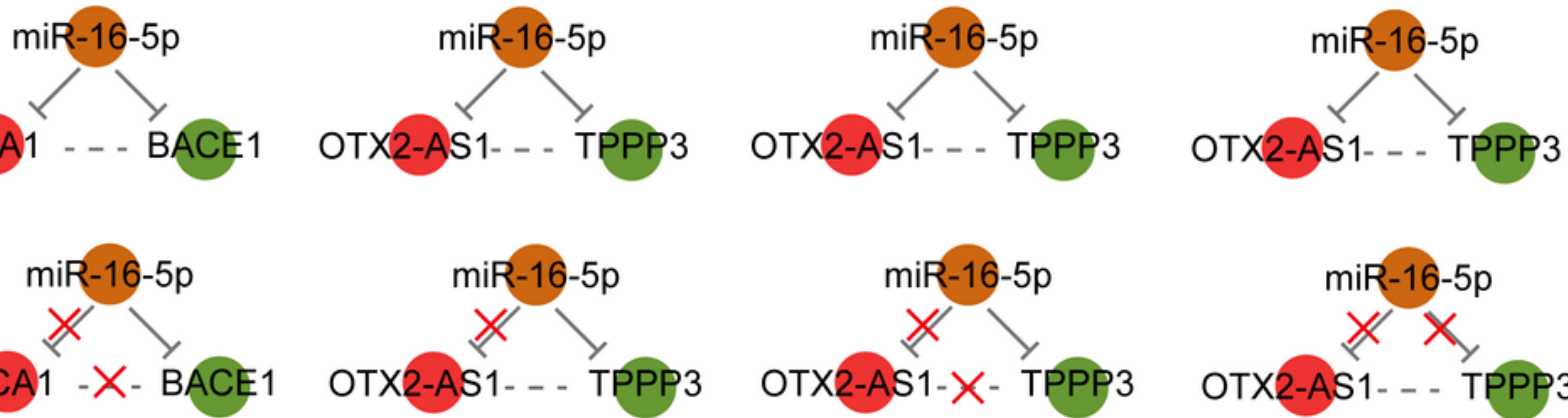
miR-16-5p
miR-16-5p
OTX2-AS1-X- TPPP3
OTX2-AS1 - - TPPP3
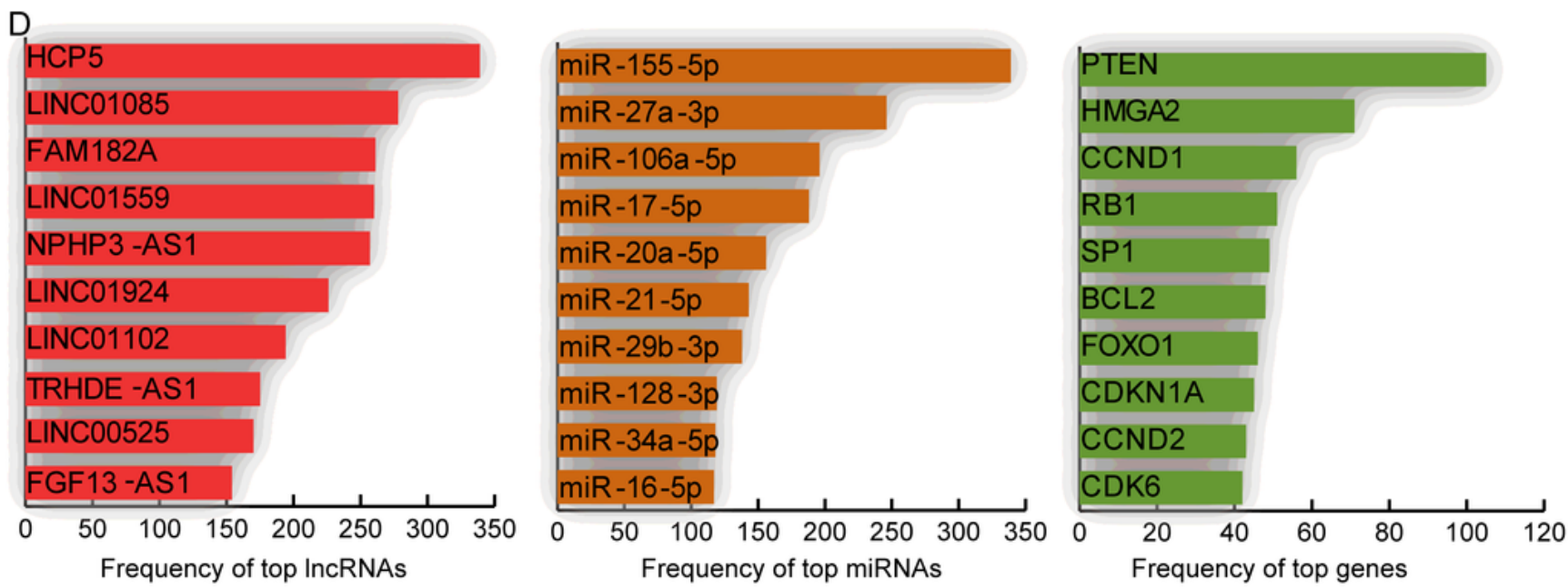

E

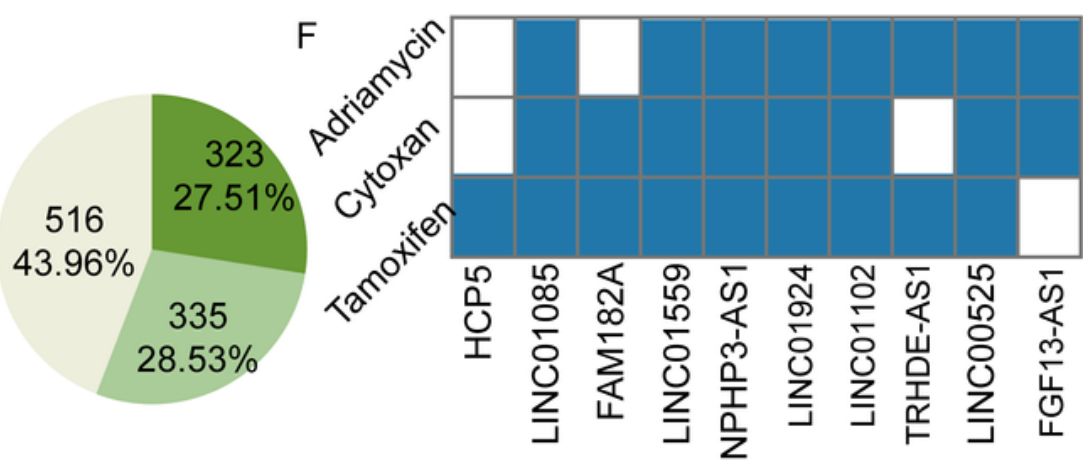

Figure 4 
Common and specific drug-resistant and -sensitive dysregulated ceRNAs in BRCA. (A) Pie charts showing the drug-resistant (left) and -sensitive (right) dysregulated ceRNAs in one, two, or all three types of drugs. (B) Drug-resistant dysregulated ceRNAs in the three diverse types of drugs. (C) Examples of the dysregulated patterns among drug response-related ceRNAs in the different types of drugs. (D) Bar plots showing the frequencies of common IncRNAs, genes, and miRNAs in drug response-related dysregulated ceRNAs. (E) Pie charts showing the dysregulated IncRNAs, miRNAs, and genes in one, two, or all three types of drugs. (F) A heat map showing the top dysregulated IncRNAs in the three diverse drug types.

A

glomerular epithelial cell differentiation regulation of biosynthetic process response to gonadotropin polyphosphate metabolic process regulation of inositol phosphate biosynthetic process positive regulation of inositol phosphate biosynthetic process negative regulation of transporter activity reproductive system development microglial cell activation cellular response to gonadotropin stimulus

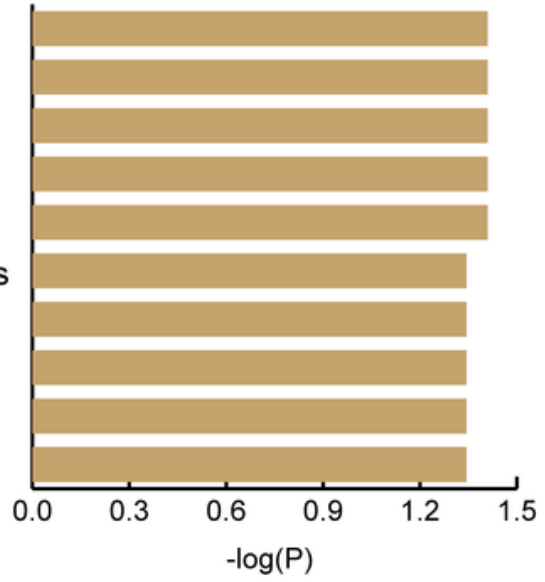

B

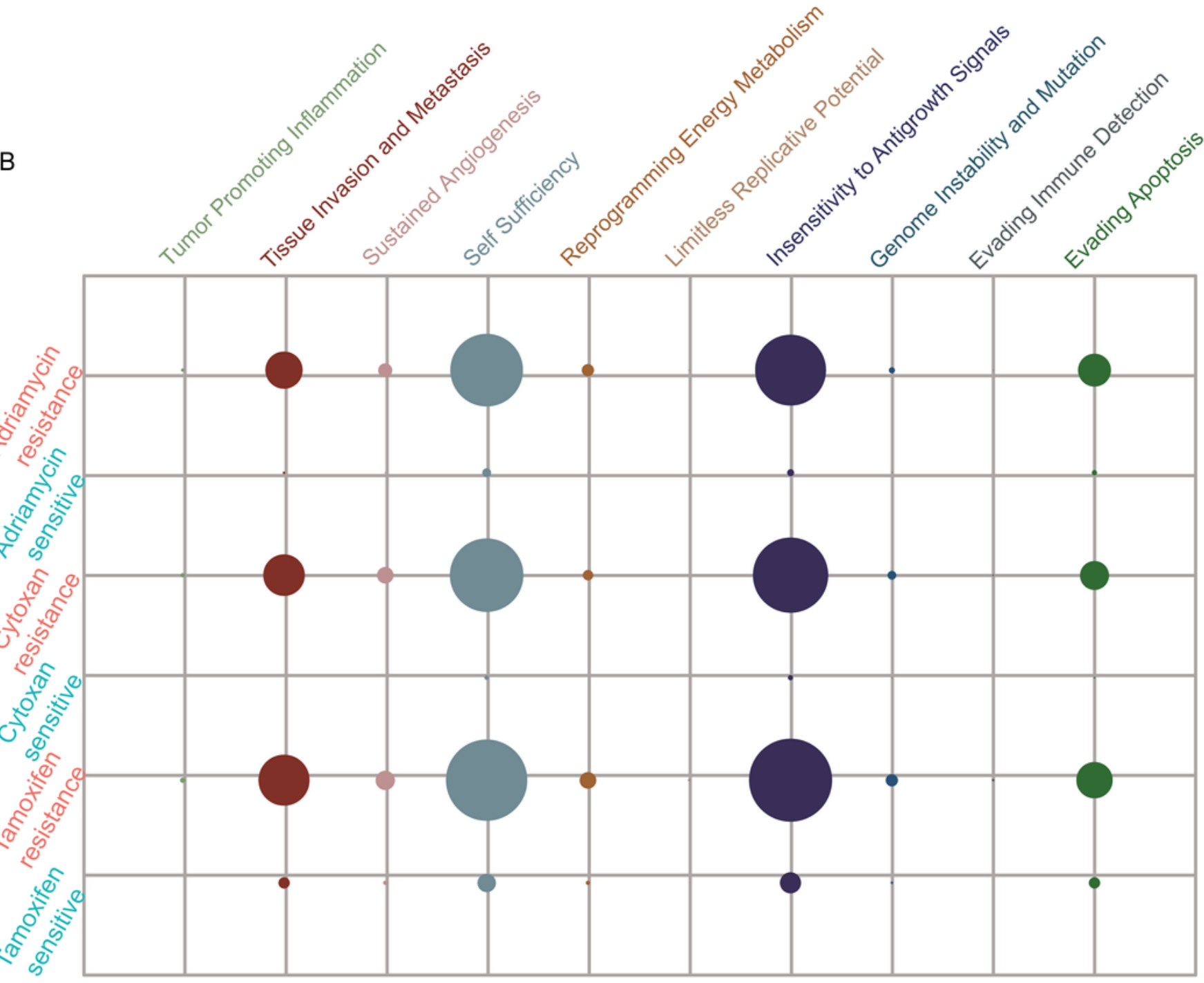


Figure 5

Cancer hallmarks in drug-resistant and -sensitive dysregulated ceRNAs. (A) A bar plot showing the significant $\mathrm{GO}$ enrichment terms, which are ranked by -log (P). (B) Circle plots showing the associations between drug response-related ceRNAs and cancer hallmarks. A larger size represents more enriched ceRNAs.

A

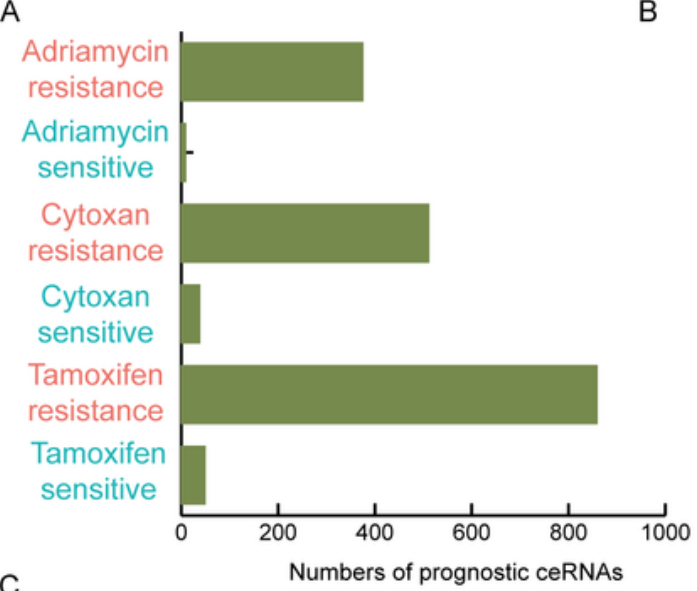

B

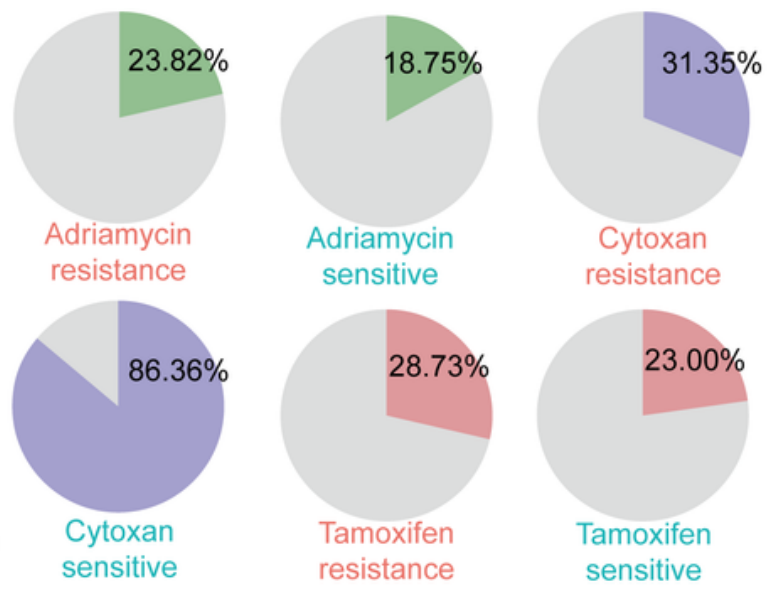

MTDH/LINC00092/miR-542-3p. KPNA4/LINC00906/miR-181b-5p MMP15/LINC00486/miR-29c-3p NFIA/LINC00906/miR-181b-5p. NRAS/LINC00906/miR-181a-5p. ITGA5/LINC01777/miR-205-5p. TCF4/LINC00906/miR-181a-5p TGFB2/LINC01777/miR-148a-3p. CYR61/LINC01777/miR-205-5p DKK2/LINC01565/miR-27a-3p. WNT4/PCAT4/miR-24-3p BCL2/LINC00906/miR-181b-5p KLF4/FMR1-AS1/miR-34a-5p TGFB3/PCAT4/miR-29b-3p SLC2A4/LINC00906/miR-106b-5p PTPN11/LINC00906/miR-181a-5p NR4A2/LINC01102/miR-34a-5p RBMXL1/LINC02120/miR-421. MDM4/LINC01102/miR-34a-5p PURA/LINC02120/miR-15a-5p
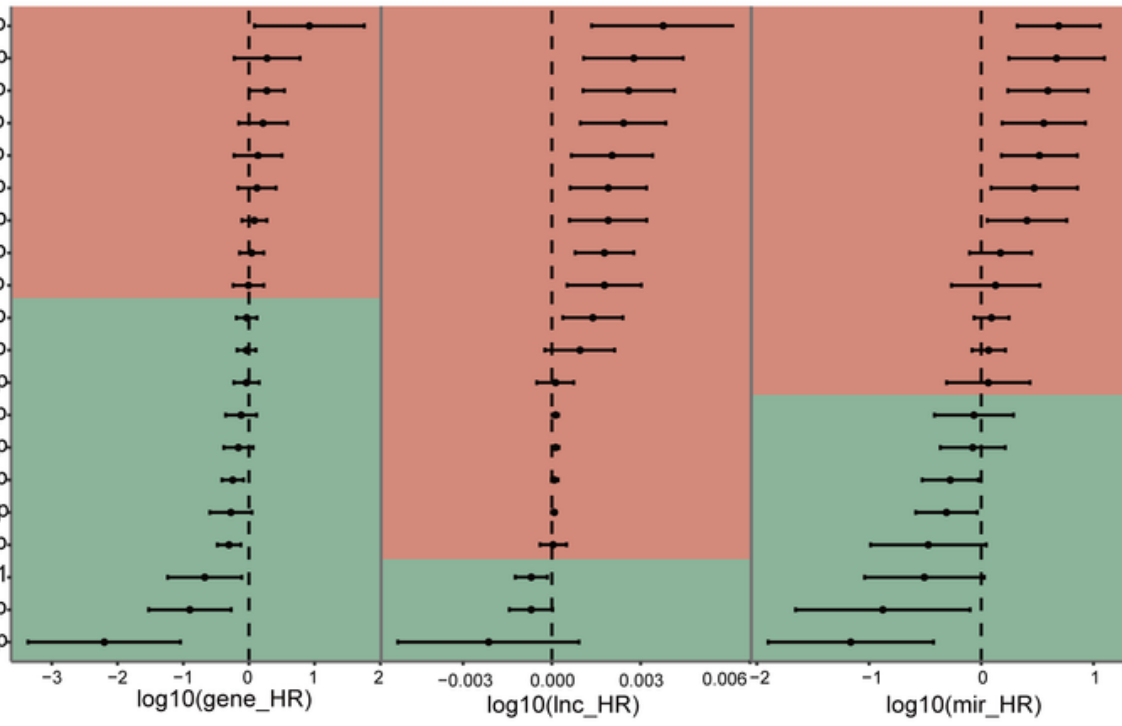

D PURA/LINC02120/miR-15a-5p
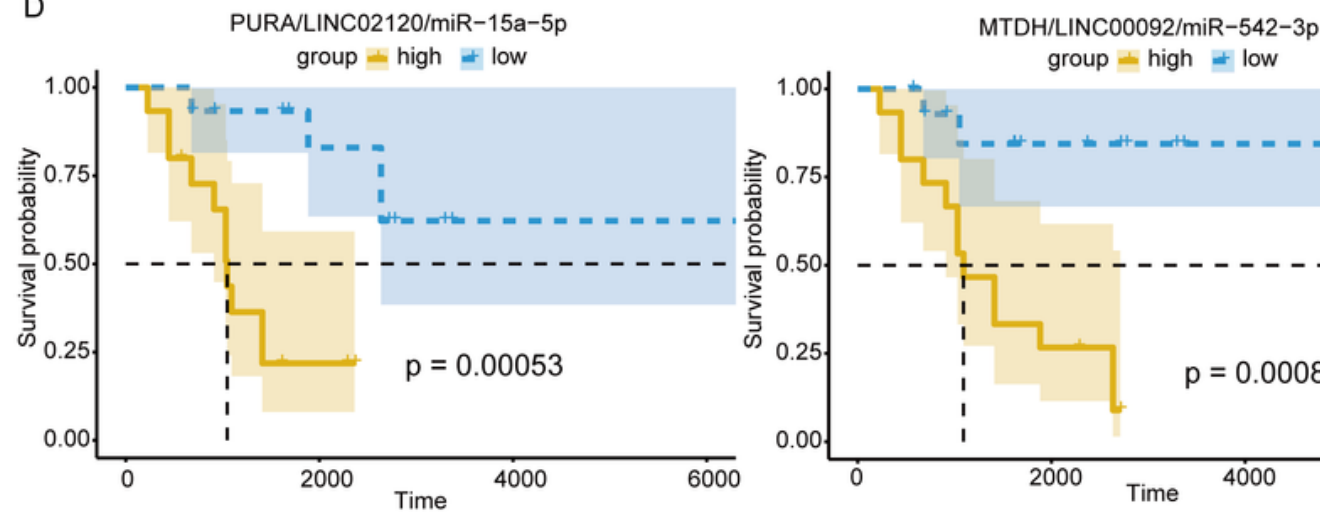

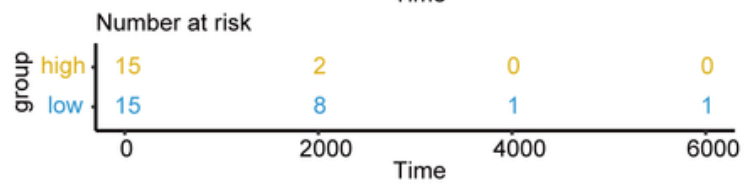

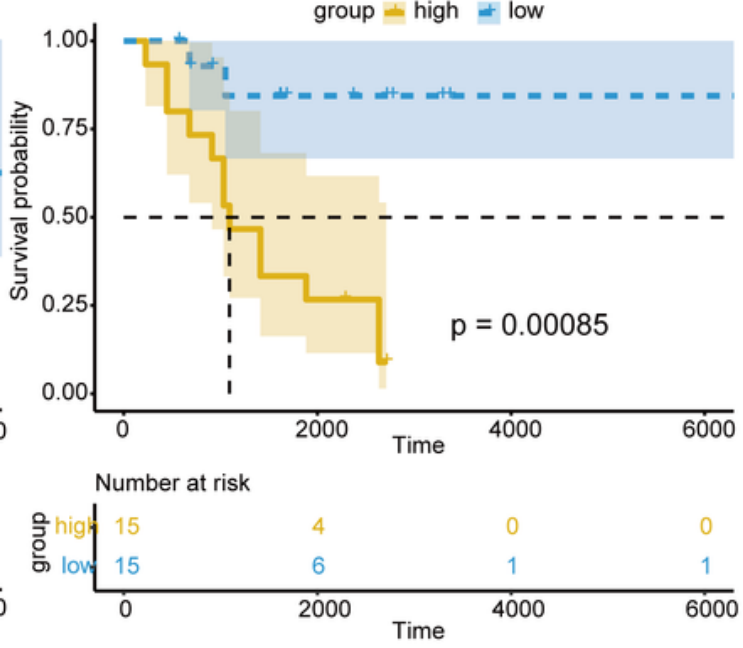

Figure 6 
Efficacy of drug-resistant and -sensitive dysregulated ceRNAs as prognostic biomarkers in BRCA. (A) A bar plot showing the numbers of prognostic drug response-related dysregulated ceRNAs. (B) Pie charts showing the percentages of prognostic drug response-related dysregulated ceRNAs. (C) HR values of the genes, IncRNAs, and miRNAs in several key prognostic drug response-related dysregulated ceRNAs. (D) Survival curves for two prognostic drug response-related dysregulated ceRNAs. Blue and yellow represent low- and high-risk groups, respectively. 